\title{
Time-Frequency Method Based Activation Detection in Functional MRI Time-Series
}

\author{
Arun $\operatorname{Kumar}^{1,2}$ and Jagath C. Rajapakse ${ }^{1,3}$ \\ ${ }^{1}$ School of Computing, NTU, Singapore \\ ${ }^{2}$ School of EEE,Singapore Polytechnic, Singapore \\ ${ }^{3}$ Biological Engineering Division, MIT, Cambridge, USA
}

\begin{abstract}
A time-frequency method based on Cohen's class of distribution is proposed for analysis of functional magnetic resonance imaging (fMRI) data and to detect activation in the brain regions. The RihaczekMargenau distribution among the various distributions of Cohen's class produces the least amount of cross products and is used here for calculating the spectrum of fMRI time-series. This method also does not suffer from the time and frequency resolution trade-off which is inherent in short-term Fourier transform (STFT). Other than detecting activation, the time-frequency analysis is also capable of providing us with more details about the non-stationarity in fMRI data, which can be used for clustering the data into various brain states. The results of brain activation detection with this techniques are presented here and are compared with other prevalent techniques.
\end{abstract}

\section{Introduction}

Human brain is a complex organ anatomically and more so in terms of its functionality. A number of signal and image based techniques have been used to understand the functionality of the brain. Functional Magnetic Resonance Imaging (fMRI) is one such imaging technique which is also non-invasive. It effectively captures the changes in the Blood Oxygenation Level Dependent (BOLD) contrast, allowing the evaluation of brain activity due to external stimuli [1. Usually, fMRI data consists of time-series emanating from each brain voxel, collected over the periods of activation and rest. The low signal-to-noise ratio (SNR) of fMRI data makes detection of the activation-related signal changes difficult; hence most of the data is collected from periodic stimulation alternating with the rest condition. The temporal dynamics of the activation response, which is delayed and is relatively slow compared to actual brain activity, is another problem that must be dealt with during analysis. Most of the present methods rely on exclusive modeling of the hemodynamic response function to detect this delayed activation 2. The most extensively used fMR data analysis techniques are variants of general linear model based on $t$-test, $F$-test, correlation coefficients (between observed responses and stimulus function) or multiple linear regression. These techniques require accurate knowledge of stimulus function [3].

J.C. Rajapakse, B. Schmidt, and G. Volkert (Eds.): PRIB 2007, LNBI 4774, pp. 368 377, 2007.

(C) Springer-Verlag Berlin Heidelberg 2007 
The frequency-domain analysis methods are able to overcome some of these shortcomings, as they do not require exclusive modeling of the hemodynamic response function and also the accurate knowledge of stimulus signal is not necessary. Spectral analysis methods have also been found to obtain better statistical estimators for short data segments 4. Fourier transform has been used traditionally to calculate the signal spectrum but in the process of calculating the spectrum, it looses the signal time information. Short-time Fourier transform (STFT), or spectrogram, is able to provide the time-based spectrum, but suffers from the time and frequency resolution trade-off condition. A number of techniques have been developed to overcome this shortcoming of STFT. The first one of these time-frequency techniques were Wigner-Ville distribution based on the autocorrelation function. This technique although overcomes the time and frequency resolution trade-off condition, it suffers from the presence of cross terms in the spectrum [5]. Various distributions in the Cohen's class aim at reducing the cross-talk, while keeping the advantages of Wigner-Ville distribution [6]. The Rihaczek-Margenau distribution of Cohen's class was found to produce the least amount of cross products and is used here for calculating the spectrum of fMRI time-series and hence for activation detection.

\section{Methods}

\subsection{Functional MRI Time Series}

Functional magnetic resonance imaging data consists of a series of threedimensional brain scans taken at regular intervals during an experiment. This complete set of spatio-temporal data can be considered as a functional image of the brain. A functional image $F$ can hence be defined as $F: \Omega \times \Theta \rightarrow$ $\{0,1 \ldots 32767\}$, where $\Omega \in N^{3}$ denotes the three-dimensional spatial domain of image voxels and $\Theta$ represents the scanning times. As these scans are acquired at regular intervals of time, $\Theta=\{\Delta, 2 \Delta \ldots n \Delta\}$ where $\Delta$ denotes the scanning interval and $n$ the total number of brain scans. A functional time-series is further defined as the functional image at a particular voxel over the experimental duration. Consider an fMRI experiment with series of brain scans taken at regular intervals; let the stimulus signal be denoted by and the change in the BOLD signal at a voxel $i$ or the mean-corrected fMRI time-series be represented by.

The Wigner-Ville and other distributions of Cohen's class use the approach of calculating power spectrum from autocorrelation function, as is used in calculation of power spectral density (psd) [7]. In the standard autocorrelation function, summation is carried out over time as shown in Eq. (11), resulting in the autocorrelation function $r_{i}(\tau)$ which is a function of lag/time-shift $\tau$ only.

$$
r_{i}(\tau)=\sum_{t \in \Theta} y_{i, t} y_{i, t+\tau}
$$

The Wigner-Ville and other distributions of Cohen's class use a variation of the autocorrelation function where time remains in the result. In this case also, the 
comparison of waveform with itself is carried over all lag values, but instead of integrating over time, comparison is done over all possible values of time. This results in so-called instantaneous autocorrelation $R_{i}(t, \tau)$ of the fMRI data as shown in Eq. (2).

$$
R_{i}(t, \tau)=y_{i, t+\tau} y_{i, t-\tau}^{*}
$$

where $\tau$ is the time lag and ${ }^{*}$ represents the complex conjugate of the signal. The instantaneous autocorrelation function retains both the lag and time value. The Fourier transform of $R_{i}(t, \tau)$ is taken along the $\tau$ dimension, hence the result is a function of both time and frequency. The relationship for determining the timefrequency distribution of Cohen's class from the instantaneous autocorrelation function is as given in Eq. (3).

$$
\rho(t, f)=\sum_{\tau=0}^{(n-1) \Delta} R_{i}(t, \tau) G(t, \tau) e^{-j 2 \pi f \tau} ; \forall t \in \Theta, f \in \mathbf{f}_{i}
$$

where function $G(t, \tau)$ is based on a two-dimensional filter (filter for the autocorrelation function) and this filter is what distinguishes various distributions within the Cohen's class, as described by Semmlow [6]. The expression for filter $G(t, \tau)$ is given by the Eq. (4/5) below for both the Wigner-Ville and RihaczekMargenau distributions of Cohen's class.

$$
\begin{gathered}
G(t, \tau)=\int_{-\infty}^{\infty} g(v, \tau) e^{j \pi v t} d v \\
g(v, \tau)= \begin{cases}1 & ; \text { for Wigner-Ville distribution } \\
e^{j v \tau / 2} & ; \text { for Rihaczek-Margenau distribution }\end{cases}
\end{gathered}
$$

Wigner-Ville distribution can be considered as a special class of Cohen's distribution as it does not apply a filter i.e. $g(v, \tau)=1$ and hence is simplified as in Eq. (6).

$$
W\left(t, \mathbf{f}_{i}\right)=\operatorname{FFT}\left(R_{i}(t, \tau)\right)
$$

The main problem with Wigner-Ville distribution is the presence of cross products in the spectrum and various distributions in the Cohen's class aim to reduce the amplitude of these cross products. All the transformations in Cohen's class of distribution produce better results when applied to a modified version of the waveform termed the Analytic signal, which is a complex version of the original signal. As the analytic signal does not contain negative frequencies, its use reduces the number of cross products. The approach based on Hilbert transform of signal, as described by Semmlow [6], is being used here to derive the analytic function. The Rihaczek-Margenau distribution of Cohen's class was found to produce the least amount of cross products and is used here for calculating the spectrum of fMRI time-series and hence for activation detection [5]. The original spectrum for Rihaczek-Margenau distribution is three dimensional as it is the time-frequency distribution with amplitude of spectral components as the third dimension. For activation detection, spectral magnitude at stimulus frequency 
can be obtained by summing together the spectral magnitude at this frequency over all time-points. The comparison of magnitude at stimulus frequency hence is used to select the set of activated voxels. The complete three dimensional time-frequency information belonging to these activated voxels can then be used for clustering and in describing the various brain states. The time-frequency data can also be used to study the variation of characteristics of hemodynamic response over different brain regions.

\section{Results and Discussion}

The proposed approach was tested on both synthetic and real functional MRI time-series and the activation detection results were compared with the statistical parametric mapping (SPM) method.

\subsection{Synthetic Data}

A two-dimensional dataset with $64 \times 64$ pixels per image scan was generated for the synthetic functional time-series, with 5 cycles of eight rest samples followed by eight task samples. The duration between two scans was taken to be two seconds $(\mathrm{RT}=2 \mathrm{~s})$ and the box-car time-series was designed for activated pixels while inactive pixels remain unchanged over time. The response of the activated pixels was then generated by convolving the box-car time-series with a gamma hemodynamic response function ( $\mathrm{lag}=5 \mathrm{~s}$ and dispersion $=6 \mathrm{~s}$ ). Independent and identically distributed (i.i.d.) Gaussian random noises was then added to the time-series of both activated and inactive pixels. Pixel intensities of image scans are given by the synthetic functional time-series (see Fig. 1). The signalto-noise ratio (SNR) is defined as $S N R=h^{2} / \sigma^{2}$, where $h$ is the amplitude of the box-car time-series, and $\sigma$ is the standard deviation of the noise. Two different values of $\mathrm{SNR}=1.2$ and $\mathrm{SNR}=2.0$ were used in generation of two sets of synthetic data.

The results obtained for the SPM ( F-test) and those obtained from RihaczekMargenau distribution based spectrum, for synthetic images with $\mathrm{SNR}=1.2$ and $\mathrm{SNR}=2.0$ are as shown in the Fig. 2 and Fig. 3 respecively.

The ROC curves obtained for two sets of synthetic data with $\mathrm{SNR}=1.2$ and $\mathrm{SNR}=2.0$ are shown in Fig. 4(a) and ROC curves for various number of epochs considered in the synthetic data are shown in Fig. 4(b). Both the plots indicate better performance for Rihaczek-Margenau (Cohen's class of distribution) spectrum based activation detection, as compared to the SPM.

\subsection{Functional MRI Data}

FMR images analyzed in this section were obtained on a 3.0 Tesla Medspec 30/100 scanner (Bruker Medizintechnik GmbH, Ettlingen, Germany) at the MRI Centre of the Max-Planck-Institute of Cognitive Neuroscience. A visualstimulation experiment using a FLASH Protocol was carried out to obtain these 


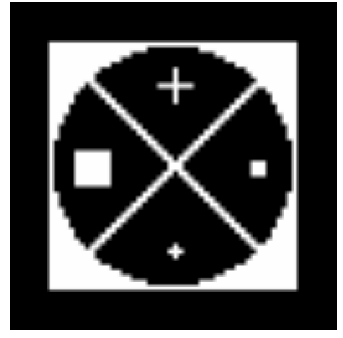

(a)

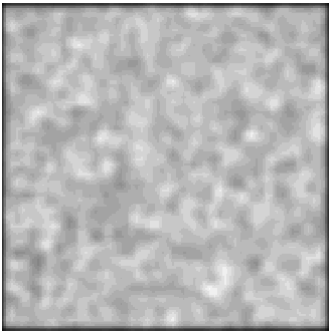

(b)

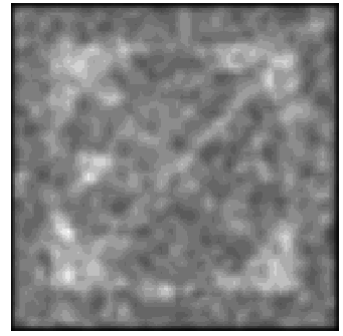

(c)

Fig. 1. Synthetic functional images $(\mathrm{SNR}=1.2)$ with i.i.d. Gaussian noises (a) Actual activation (b) representative scan of rest state (40th scan) (c) representative scan of stimulus state (80th scan).

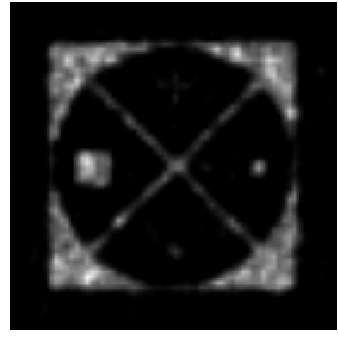

(a)

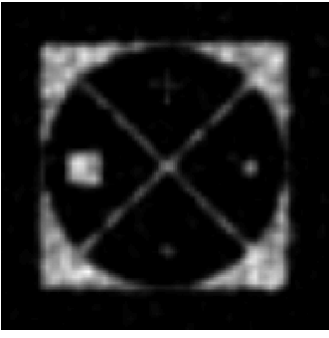

(b)

Fig. 2. Illustration of the detected activation on synthetic data with independent noise $(\mathrm{SNR}=1.2)$ based on (a) SPM (F-test) (b) Rihaczek-Margenau distribution spectrum

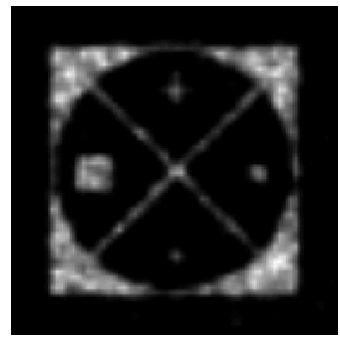

(a)

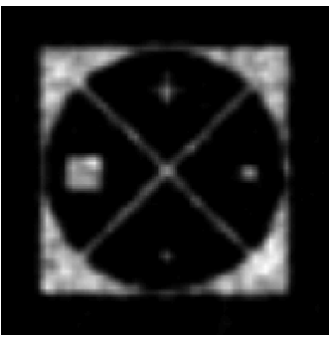

(b)

Fig. 3. Illustration of the detected activation on synthetic data with independent noise $(\mathrm{SNR}=2.0)$ based on (a) SPM (F-test) (b) Rihaczek-Margenau distribution spectrum 


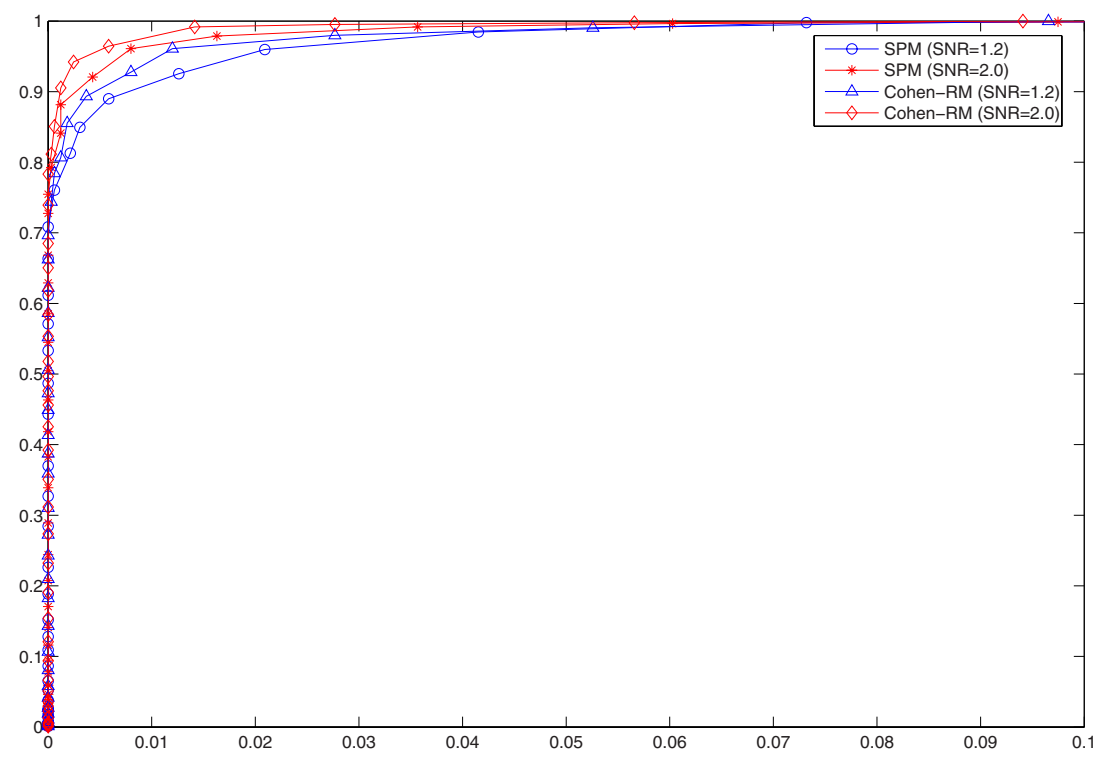

(a)

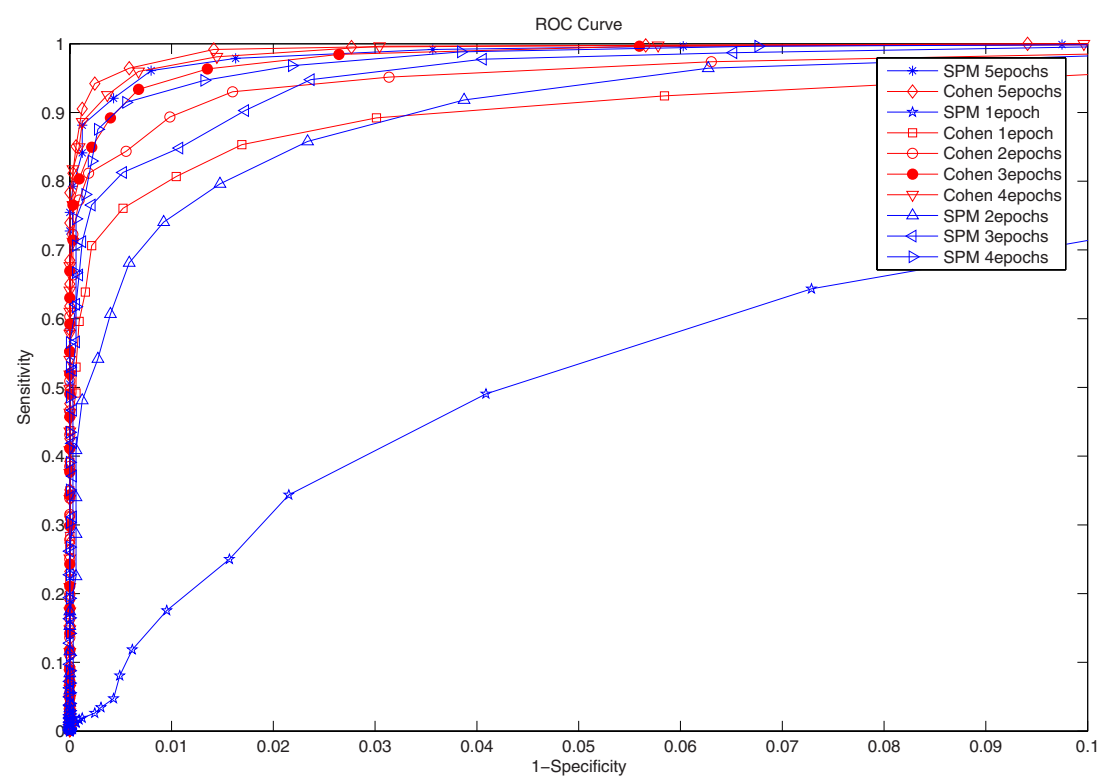

(b)

Fig. 4. ROC curves for synthetic data comparing the Rihaczek-Margenau distribution and SPM (F-test) technique considering (a) multiple epochs with varying SNR $(\mathrm{SNR}=1.2$ and $\mathrm{SNR}=2.0)$ (b) varying number of epochs with constant SNR. 


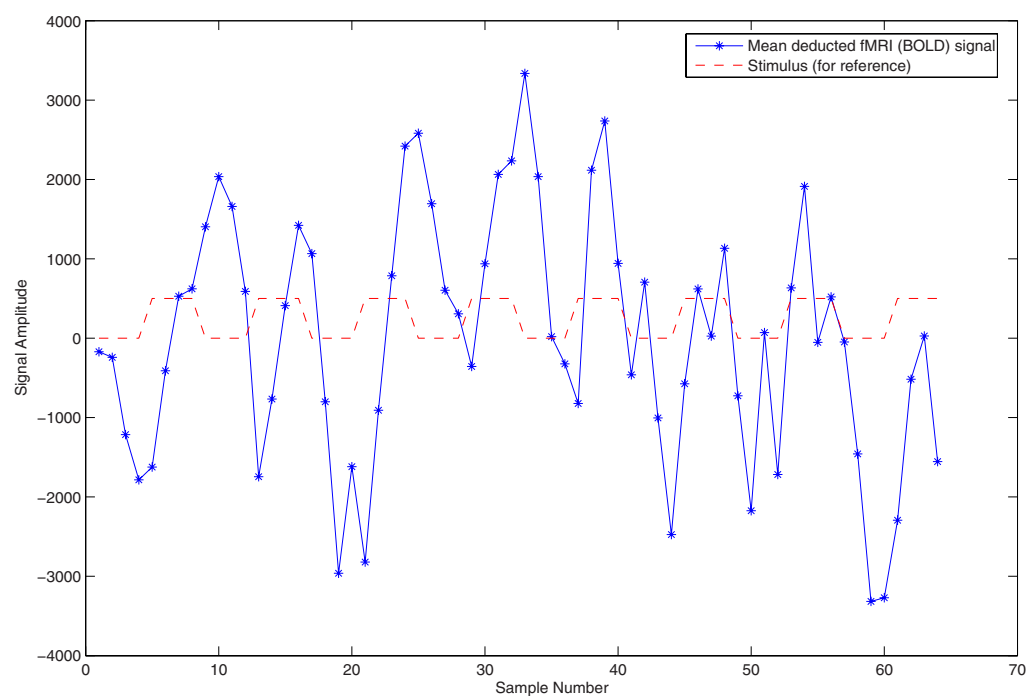

Fig. 5. Illustration of noisy fMRI data from a single voxel with respect to the stimulus signal

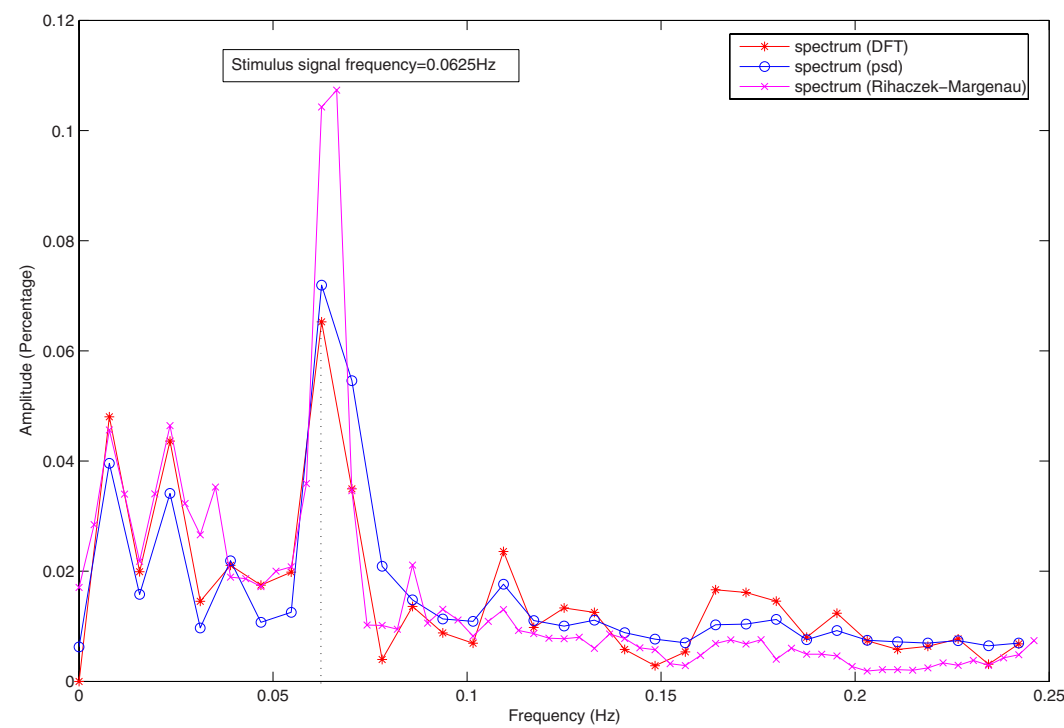

Fig. 6. Comparison of spectrum based on psd, discrete Fourier transform and RihaczekMargenau distribution of Cohen's class for the noisy voxel data (Fig. 5) 


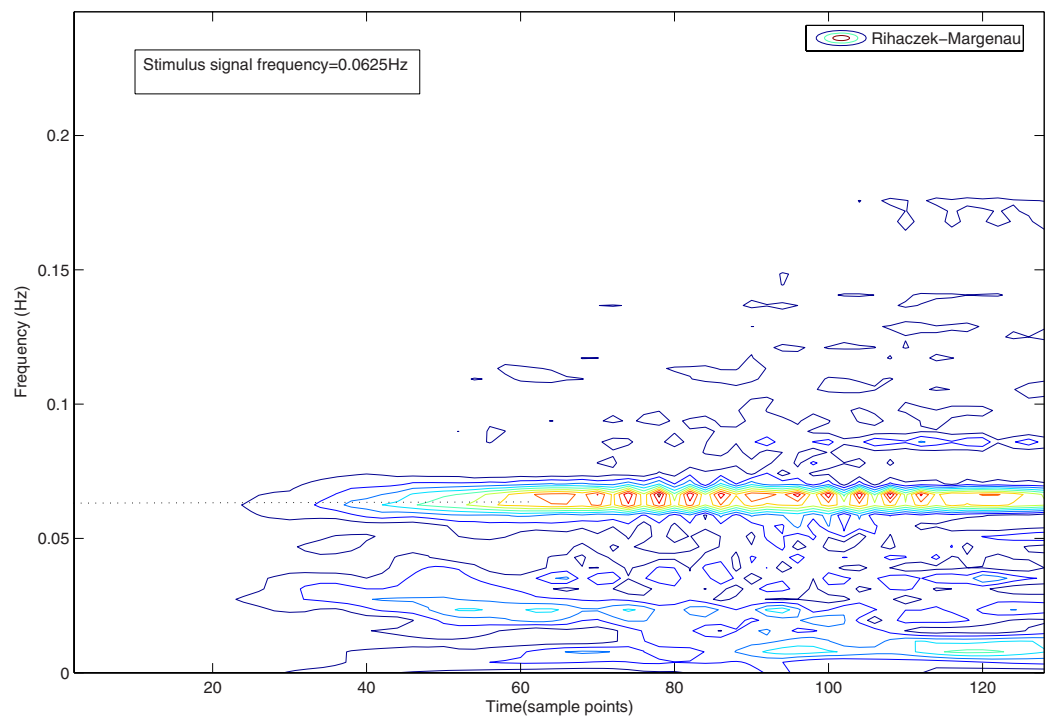

(a)

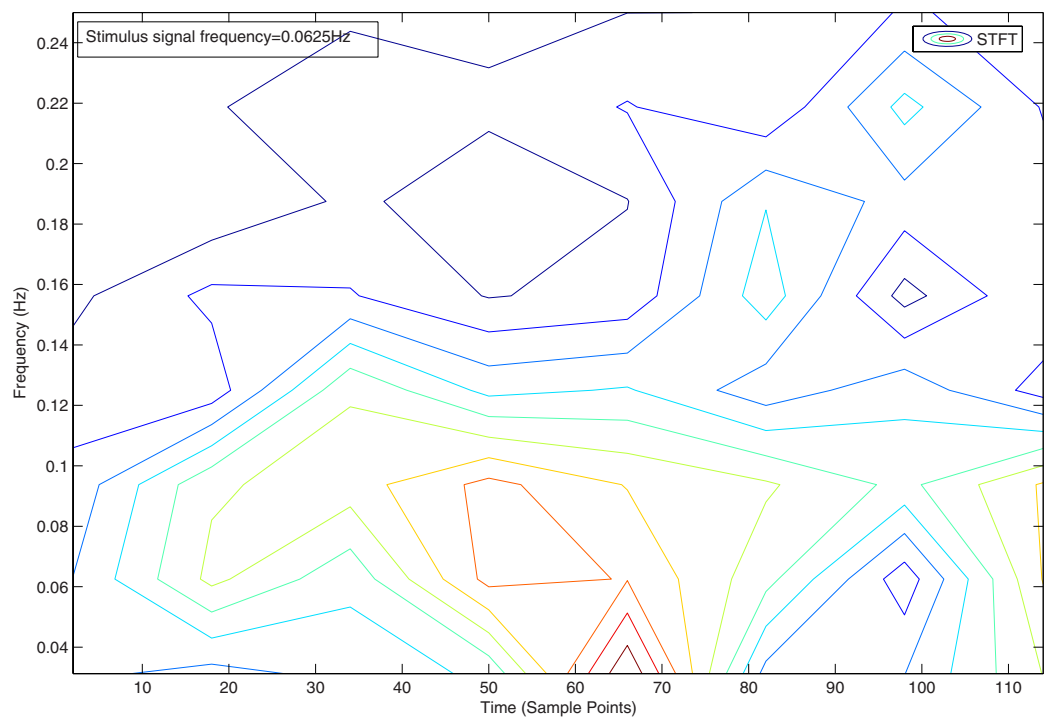

(b)

Fig. 7. Contour plot of noisy voxel data of Fig. 5 based on (a) Rihaczek-Margenau distribution (b) Short time Fourier transform (window size of 16 samples and overlap of 8 ) 
images $(\mathrm{TR}=80.5 \mathrm{~ms} ; \mathrm{TE}=40 \mathrm{~ms}$; matrix $=128 \times 64$; The image matrices were zero-filled to obtain $128 \times 128$ images with a spatial resolution of $1.953 \times 1.953$ $\mathrm{mm}$; slice thickness $=5 \mathrm{~mm}$ and $2 \mathrm{~mm}$ gap). In all the experiments, on and off stimuli were presented at a rate of $\mathrm{RT}=5.16 \mathrm{~s}$ per sample. Each stimulation period had four successive stimulated, ON, scans followed by four rest scans, i.e., stimulation OFF scans. Further details of this experiment can be found in 8 .

The spectrum as obtained from Rihaczek-Margenau distribution for a noisy voxel data (Fig. 5) is compared with the spectrum obtained from the DFT and psd in the Fig. 6. It is clear from the figure that the spectrum obtained with Rihaczek-Margenau distribution has comparatively higher magnitude at stimulus frequency and much lower magnitude at noise frequencies as compared to the psd and DFT spectrum.

The original spectrum for Rihaczek-Margenau distribution is three dimensional as it is the time-frequency distribution with amplitude of spectral components as the third dimension (Fig. 7) and the two-dimensional plot (Fig. 6) has been obtained by summing together the spectral magnitude for a given frequency at all time-points. The comparison of magnitude at stimulus frequency hence is used to select the set of activated voxels.

Activation detection results for the multiple cycle visual task fMRI data for Rihaczek-Margenau distribution and SPM (F-test) are shown in the Fig. 8 ,

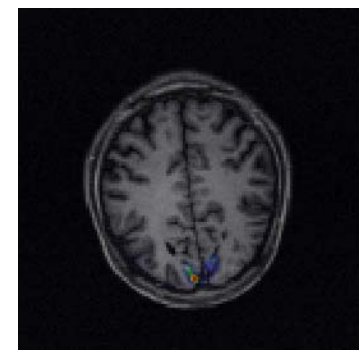

(a)

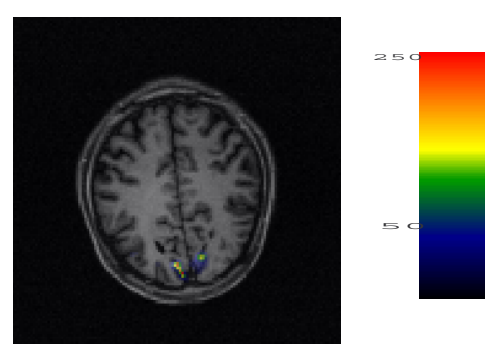

(b)

Fig. 8. Illustration of activation detection using (a) Rihaczek-Margenau distribution (b) SPM (F-test)

\section{Conclusion}

A time-frequency method based on Cohen's class of distribution for spectrum calculation and detection of activated voxels in fMRI data was proposed and presented in this paper. The technique is found to produce more accurate activation maps as compared to the existing techniques. The technique is robust to uncorrelated noise and is also able to detect voxels with delayed activation. Our technique does not require prior information of the HRF, also the precise information of stimulus signal is not required. The information from the 
time-frequency analysis has not been exploited fully as yet in the present paper and will be used in studying the variation of characteristics of hemodynamic response over different brain regions and also in describing the various brain states in our future work. Our future work also includes incorporation of variation of structures and tissues into the detection of brain activation in multi-modality frameworks 9 .

\section{References}

1. Farckowiak, R.S.J., Friston, K.J., Frith, C.D., Dolan, R.J., Mazziota, J.C.: Human Brain Function. Academic Press, USA (1997)

2. Friston, K.J., Jezzard, P., Jezzard, T.R.: Analysis of functional MRI time-series. Human Brain Mapping 1, 153-171 (1994)

3. Friston, K.J., Holmes, A.P., Worsley, K.J., et al.: Statistical Parametric Maps in Functional Imaging: A General Linear Approach. Human Brain Mapping 2, 189 210 (1995)

4. Mitra, P.P., Pesaran, B.: Analysis of Dynamic Brain Imaging Data. Biophysical Journal 76, 691-708 (1999)

5. Cohen, L.: Time-Frequency Analysis. Prentice Hall, Englewood Cliffs, NJ (1995)

6. Semmlow, J.L.: Biosignal and biomedical image processing. Marcel Dekker Inc., USA (2004)

7. Kumar, A., Rajapakse, J.C.: Power spectral based detection of brain activation. Neural Computing and Applications (accepted March 2, 2007) (in print)

8. Rajapakse, J.C., Kruggel, F., Maisog, J.M., Cramon, D.Y.: Modeling Hemodynamic Response for Analysis of Functional MRI Time-Series. Human Brain Mapping 6, 283-300 (1998)

9. Zhou, J., Rajapakse, J.C.: Segmentation of subcortical brain structures using fuzzy templates. Neuroimage 28(4), 927-936 (2005) 